\title{
La représentation du corps féminin dans Anatomie de l'enfer de Catherine Breillat
}

\author{
Fabien DEMANGEOT \\ Université de Bourgogne, Université Paris 1 \\ demangeot.fabien@orange.fr
}

Recibido: $15 / 11 / 2015$

Aceptado: 20/05/2016

\begin{abstract}
Résumé
Cette étude interrogera, à travers l'exemple du film Anatomie de l'enfer, la manière dont Catherine Breillat, considérée comme l'une des cinéastes les plus controversées de notre époque, met en scène le corps féminin. En réinvestissant certains mythes archaïques tout en jouant avec les codes de la pornographie contemporaine, la réalisatrice de Romance dépeint une féminité aussi torturée que désabusée. Notre analyse mettra en exergue le caractère ambigu de cette représentation du corps féminin perpétuellement ouvert au jeu des interprétations.
\end{abstract}

Mots clé : Catherine Breillat, corps, sexualité, ambiguïé, pornographie, féminité, féminisme.

\section{La representación del cuerpo femenino en Anatomie de l'enfer de Catherine Breillat}

\section{Resumen}

Este estudio analiza, a través del ejemplo de la película Anatomie de l'enfer, la manera en la que Catherine Breillat, considerada una de las cineastas más controvertidas de nuestro tiempo, presenta el cuerpo femenino. Al reinvertir algunos mitos arcaicos mientras juega con los códigos de la pornografía contemporánea, la directora de Romance retrata la femineidad tan torturada como desilusionada. Nuestro análisis pondrá de relieve la naturaleza ambigua de esta representación del cuerpo femenino perpetuamente abierto al juego de las interpretaciones.

Palabras clave: Catherine Breillat, el cuerpo, la sexualidad, la ambigüedad, la pornografía, la feminidad, el feminismo.

\section{The Representation of the Female Body in Anatomie de l'enfer by Catherine Breillat}

\begin{abstract}
Using the film Anatomie de l'enfer as a paradigmatic example, this study examines the ways in which Catherine Breillat — one of the most controversial filmmakers of our time-represents the female body. By recasting some ancient myths while playing with the codes of contemporary pornography, the director of Romance portrays femininity as a tortured and disillusioned identity. This analysis will
\end{abstract}


highlight the ambiguous nature of this representation of the female body, perpetually open to the play of interpretations.

Keywords: Catherine Breillat, body, sexuality, ambiguity, pornography, femininity, feminism.

\section{Referencia normalizada}

Demangeot, F. (2016). «La représentation du corps féminin dans Anatomie de l'enfer de Catherine Breillat ». Thélème. Revista Complutense de Estudios Franceses, Vol. 31, Núm. 2 : 259-270. http://dx.doi.org/10.5209/rev_THEL.2016.v31.n2.51055

Cinéaste à la réputation sulfureuse, Catherine Breillat a basé l'ensemble de son œuvre sur la question de la sexualité féminine. Loin de toute revendication féministe, elle n'a cessé de mettre en scène le corps de la femme à la fois comme un objet de désir et de répulsion. Dans son premier film, Une vraie jeune fille, en 1975, la réalisatrice de Romance s'intéressait déjà à l'ambiguïté de ce corps qui, bien que tentateur, ne cesse de suinter et de suppurer. Excitée par l'idée d'explorer ce corps qu'elle considère comme obscène, Alice, l'adolescente du film de Breillat, découvre progressivement que le dégoût peut aussi être synonyme d'érotisme. La matière devient même un support masturbatoire comme l'atteste la scène au cours de laquelle la jeune fille frotte longuement son pied sur le sol sale et humide des toilettes du pensionnat. Libre au spectateur d'imaginer ce que la flaque dans laquelle Alice trempe son pied peut bien contenir même si Breillat, en multipliant, tout au long de son film, les analogies de type sexuel autour du sperme et des menstruations, invite forcément à la métaphore. Chez la réalisatrice de Parfait amour !, c'est bien le corps dans son intégralité qui est au centre de la représentation. Les sécrétions, comme les organes sexuels, sont exposés aux yeux d'un spectateur souvent peu habitué à voir ce type d'images au cinéma. Bien que tous les films de la réalisatrice ne contiennent pas d'inserts pornographiques, ils exposent des corps féminins en souffrance pour qui la sexualité est moins source de plaisir que d'angoisse. Cette ambiguité trouve la plus belle de ses illustrations dans l'avant dernier long-métrage de la réalisatrice, le controversé Anatomie de l'enfer.

En 2004, Anatomie de l'enfer marque un véritable tournant dans la carrière de la cinéaste. Plus cérébrale que jamais, la réalisatrice interroge, dans ce film jugé scandaleux, le rapport que la femme entretient avec son propre corps. Dans un lieu hors du monde, un homme et une femme s'affrontent pour mieux s'unir dans leur dégoût commun de l'anatomie féminine. À travers le personnage anonyme interprété par Amira Casar, Breillat convoque l'imagerie archaïque de la femme pécheresse. Depuis la nuit des temps, la femme est celle qui porte le poids de la culpabilité. Si son corps saigne, c'est parce qu'elle paye la faute d'Ève qui, en croquant dans la pomme, a désobéi à Dieu. Dans l'Ancien testament, le sang des règles est le signe de l'impureté féminine. Comparée à la gonorrhée, dans Le Lévitique, les menstruations ont longtemps été considérées comme une blessure sexuelle. Cette meurtrissure, la 
femme du film de Breillat l'expose pour mieux la combattre. Elle cherche à faire voir, à l'homme, cette autre figuration de l'altérité, ce corps qu'elle ne possède pas mais qui la possède. Le postulat de base du film, libre adaptation de La Maladie de la mort de Marguerite Duras, présente une femme qui paye un homme homosexuel pour la regarder par là où elle n'est pas regardable. Le cadre du film, qui n'est pas sans rappeler l'univers clos de Sade, que l'on songe au couvent de Sainte-Marie des Bois de Justine ou les malheurs de la vertu ou au château de Silling des 120 journées de Sodome, est déjà propice à l'introspection. Breillat dépouille, au maximum, son film de tous les artifices habituels de son cinéma. Dans un lieu hors du monde, un homme et une femme, s'affrontent pour mieux s'unir dans leur dégoût commun de l'anatomie féminine. Anatomie de l'enfer, contrairement aux autres films de Breillat, est un huisclos particulièrement verbeux où la parole sert moins les actes qu'elle ne les dicte. Le caractère performatif du langage empêche toute spontanéité. Les personnages dissertent, avant, durant et après leurs rapports sexuels. Ils cherchent, non pas à jouir de leurs corps, mais à comprendre les mécanismes du sexe féminin. Cette exploration à la fois biologique et philosophique ne s'embarrasse d'aucune forme de psychologisme. Les personnages, pareils aux innombrables victimes sadiennes, n'ont pas d'identité. Ils sont l'homme et la femme, de purs corps et de purs esprits présents dans le seul but de disserter autour de la question du corps féminin. Alors que chez Sade, les anonymes ne sont là que pour subir toutes sortes de perversions sexuelles, chez Breillat, ils se présentent comme de véritables théoriciens du corps et de la sexualité. La femme d'Anatomie de l'enfer est disséquée de l'intérieur comme de l'extérieur. Elle revendique, d'ailleurs, à plusieurs reprises, au cours du film, sa position de cobaye. Si dans les autres films de la réalisatrice, le corps de la femme était déjà exposé au regard des hommes, c'est la première fois que cette exhibition est l'objet d'un discours théorique construit autour de représentations culturelle et mythologiques. Dans La Maladie de la mort de Duras, l'homme paye la femme pour la voir nue. Le corps féminin, bien qu'exposé, n'est pas présenté ici comme un objet de curiosité scientifique. C'est l'homme, atteint de cette maladie de la mort qui donne son titre à l'ouvrage, qui souffre de sa condition et non la femme qui fait don de son corps. Néanmoins, chez Duras comme chez Breillat, le corps féminin appelle la haine et la violence. Ainsi dans La Maladie de la mort, Duras évoque un corps qui appelle l'étranglement, le viol, les mauvais traitements, les insultes, les cris de haine ainsi que le déchaînement des passions entières et mortelles (Duras, 1982: 25). Dans Anatomie de l'enfer, Breillat représente ce désir de destruction de manière beaucoup plus prégnante puisque l'homme cherche à tuer cette femme qu'il considère comme diabolique. Tout au long du film, le corps féminin ne cesse d'être violenté. La femme abandonne son corps aux désirs de l'homme qui, à son contact, se trouve dans l'incapacité de refréner ses pulsions. Cette maladie de la mort dont est atteint le personnage masculin du roman de Duras a contaminé l'héroïne d'Anatomie de l'enfer qui, parce qu'elle ne supporte plus d'être une femme, cherche, au début du film, à mettre fin à ses jours en se coupant les poignets, dans les toilettes d'une boîte de nuit gay. Habillée de blanc, symbole d'une pureté qu'elle ne retrouvera jamais, elle 
expose un corps dégradé qui fait écho aux propos de Michel Leiris qui, dans $L$ 'Âge d'homme, évoquait sa propre angoisse du sexe féminin :

\footnotetext{
J'ai couramment tendance à regarder l'organe féminin comme une chose sale ou comme une blessure, pas moins attirante pour cela, mais dangereuse en elle-même, comme tout ce qui est sanglant, muqueux, contaminé (Leiris, 1939: 31).
}

Ce lien entre fascination et répulsion est au centre du film de Breillat qui interroge les sources de cette angoisse en donnant le rôle clef de l'explorateur à un homme qui n'aime pas les femmes. En apparaissant, au début du film, comme une sorte d'âme errante qui chercherait le repos, la femme d'Anatomie de l'enfer se pose en victime pour finalement se présenter comme un véritable bourreau. L'homosexuel, loin d'être considéré comme un pécheur, incarne ici une forme d'innocence pervertie. Dans le film de Breillat, c'est la femme qui souille l'homme avec son sang impur. Rocco Siffredi, star mondialement connu du $\mathrm{X}$, incarne cet homme qui ne connaît rien à l'anatomie féminine. En pénétrant un sexe de femme sanguinolent, il découvre une altérité qui le mènera à sa propre perte. Breillat filme, en gros plan, le pénis ensanglanté de son acteur. La pénétration n'intéresse pas la cinéaste qui préfère s'attarder sur le caractère contaminé ou contaminant des sexes qu'elle expose. Pour l'homosexuel d'Anatomie de l'enfer, la femme est répugnante parce que son sexe suppurant appelle la Mort. Suite à ce coït sanguinolent, Rocco Siffredi examine longuement son appareil génital meurtri. La présence, au-dessus du lit, d'un christ en croix confère, à la scène, une certaine forme de mysticisme. L'homme est blessé dans sa chair, sans qu'aucune blessure ne soit apparente. Il craint de ne pouvoir essuyer ce sang qui, pour reprendre les propos tenus par Breillat, dans Pornocratie, est à la fois plus tendre et plus écœurant que la première exsudation de sperme sur le gland de la verge à l'instant turgescente (Breillat, 2001 : 75). Plusieurs décennies avant Breillat, Pasolini mettait déjà en scène, dans sa pièce Orgie, cette peur pathologique du sexe féminin :

Ma chair veut vraiment la mort!

Ma queue veut vraiment le sang ! (Pasolini, $1988: 46)$.

Les deux auteurs reprennent, à leur compte, le mythe du Vagina dentata qu'ils délestent cependant de son caractère moralisateur. Dans de nombreuses cultures, le mythe du Vagina dentata est véhiculée par des apologues préventifs dans le but de décourager les hommes de commettre un viol ou même simplement d'avoir des rapports sexuels avec une inconnue. Breillat s'intéresse principalement à la dimension psychanalytique de ce mythe. L'homme d'Anatomie de l'enfer craint de perdre ses attributs virils, d'être castré par le sexe impur de cette femme qu'il ne peut cependant s'empêcher de pénétrer. Il est tentant de voir, derrière les discours de Pasolini et de Breillat, une certaine forme de misogynie or les deux cinéastes interrogent, avant tout, la culture et les mythes fondateurs de la sexualité et de la féminité (Stevens, 2013: 79). En effet, en dehors de son caractère foncièrement 
impur, le sexe féminin a toujours été considéré comme un véritable « générateur de psychose » :

L'utérus est pensé non comme un organe actif/productif et symbolisant comme tel, mais comme un pur «lieu» (on parle couramment de «milieu» utérin) et, qui plus est, ainsi que nous le dit l'étymologie du mot, comme un lieu qui est «en arrière» (usteros), pré-historique, pré-natal, qui appelle et n'appelle qu'à la régression. [ ...] Ce lieu, pour qui n'en imagine rien au-delà du phallus, est fantasmé comme absolument en-deçà, comme le trou noir du continent noir et, pis encore, comme générateur de «psychose », « de bruit blanc » (Fouquette, $1995: 75)$.

Breillat réutilise toutes ces références pour mieux proposer, en dehors de toute considération morale, une nouvelle image de la sexualité féminine. Dans Anatomie de l'enfer, la cinéaste reprend les discours pornographiques traditionnels pour se lancer dans une véritable contestation idéologique de ceux-ci. Réifié, le personnage masculin d'Anatomie de l'enfer est moins sujet qu'objet. Il évoque l'homme de La Maladie de la mort qui, bien qu'en position de dominant, puisqu'il est client et non prostitué, finit par se faire asservir par la femme qu'il paye. Le fantasme de la femme soumise est déconstruit par la cinéaste qui joue avec les figures imposées du genre pornographique. Ce n'est plus l'homme, comme on a pu le voir précédemment, qui paye la femme mais la femme qui paye l'homme pour assouvir ses fantasmes. Obligé de regarder, de toucher et de pénétrer ce corps qui le rebute, l'homosexuel du film de Breillat évoque les innombrables figures de femmes soumises propres au porno hétérosexuel mainstream. L'hérö̈ne d'Anatomie de l'enfer est en position de force bien que son corps soit perpétuellement ouvert, dans l'attente d'un coït forcément déceptif. Breillat filme une sexualité exclusivement cérébrale. Si la femme jouit, c'est parce qu'elle l'a décidé et non parce que son partenaire lui a procuré du plaisir. En demandant à son observateur de la pénétrer avec un godemiché en pierre, l'héroïne d'Anatomie de l'enfer dépossède l'homme de sa propre masculinité. Elle évoque l'élasticité et la profondeur de son vagin qui absorbe l'objet, qu'il soit de pierre ou de chair, pour mieux le recracher. En jouant à enfoncer et à retirer le godemiché du sexe de sa partenaire, l'homme examine ce gouffre qu'il ne peut explorer. Filmé en gros plans, le vagin est, dès lors, présenté comme une sorte de créature monstrueuse. Il effraie autant qu'il attise la curiosité.

Breillat cherche ici à dépouiller le désir de l'homme, elle se rapproche des théories d'Angela Carter pour qui la pornographie est le symptôme d'une société phallocratique qui ne conçoit le rapport de l'homme à la femme que sur le mode de l'exploitation. Selon Frédéric Regard, auteur de L'Écriture féminine en Angleterre, Carter croit, comme Breillat, en la possibilité d'une " pornographie morale » dans laquelle la femme serait moins l'objet que le sujet de l'action (Regard, 2002 : 107). Si la femme est moins "pénétrante» que "pénétrée », elle finit par destituer l'homme de son phallus. Le sexe masculin s'effacera alors au profit de substituts sexuels pour le moins surprenants. Godemiché en pierre, bêche : les instruments de plaisirs féminins annihilent le désir de l'homme qui ne jouira jamais si l'on excepte une courte scène de fellation filmée en hors-champ. Suite à ce premier rapport sexuel, 
le visage d'Amira Casar, les lèvres couvertes de sperme, est aussi extatique que celui des innombrables figures de martyrs qui jalonnent l'œuvre de Georges Bataille à laquelle Breillat n'a cessé de faire allusion tout au long de sa carrière. Dans Histoire de l'œil, le prêtre, violé et torturé, à l'intérieur de la sacristie, semble acquérir, au cœur même de son supplice, une sorte de beauté extatique qui le rapproche de la figure du saint Sébastien peint par Guido Reni :

Une sorte de joie absurde commença à lui ouvrir la bouche, il croisa les bras sur sa poitrine nue et
nous regarda enfin avec des yeux extatiques : le martyre... un étrange état de purification était venu au
misérable et ses yeux en étaient comme illuminés (Bataille, $1993: 62$ ).

Chez Bataille, l'expérience sexuelle, surtout quand elle est dégradante, amène à un état de sainteté. La femme d'Anatomie de l'enfer, en décidant de sucer le sexe d'un inconnu, en plein milieu de la rue, semble, elle aussi, rechercher cette extase statique propre aux martyrs pervertis de Bataille. Elle s'accapare également le phallus de son partenaire qui ne se doute pas, après lui être venu en secours, qu'il va se retrouver à sa merci. Dans Anatomie de l'enfer, comme dans Histoire de l'œil, c'est la femme qui abuse de l'homme en l'obligeant, contre son gré, à devenir son objet sexuel. Cette inversion des valeurs propre au genre pornographique est dédoublée par une imagerie christique assimilant les plaies béantes du christ aux orifices sexuels ensanglantés de l'héroïne. Seul objet de décor, à l'intérieur de la chambre à coucher, le crucifix expose les souffrances du christ auquel la femme du film de Breillat ne cesse implicitement de se comparer. Comme le fils de Dieu, la femme est blessée d'une plaie éternelle. Réduite métonyquement à son seul sexe, elle perd progressivement son statut d'être humain pour incarner le malaise non pas de la civilisation mais de la condition féminine. Le sexe féminin, comparé, dans le film, au cadavre d'un oisillon écrasé, est aussi monstrueux que risible. Le magma informe, représenté par la dépouille de l'animal, lors de la scène du souvenir d'enfance, est filmé en gros plan. Il apparaît comme la représentation imagée de cette intériorité monstrueuse sur laquelle les personnages d'Anatomie de l'enfer ne cessent de fantasmer.

Selon David Vasse, auteur de Catherine Breillat, un cinéma du rite et de la transgression, le rôle de la femme est de dire sa souffrance de posséder un tel corps, à ce point de refus qu'elle se sent dépossédée d'elle-même à cause de lui et de ses propriétés presque tératologiques pendant que l'homme l'observe du haut de son ignorance (Vasse, 2004 : 126). En exhibant son corps, la femme d'Anatomie de l'enfer défend sa propre monstruosité face à un homme qui n'éprouve aucune attirance pour son sexe. Le terrain d'exploration (hétéro)sexuelle est aussi inquiétant qu'attirant pour l'homosexuel incarné par Rocco Siffredi. Le corps de la femme, devenu un véritable objet de fascination et de répulsion, correspond parfaitement ici à la définition de l'obscénité donnée par Estelle Bayon, dans son ouvrage Le cinéma obscène : 
L'obscène est un franchissement vers un autre territoire, une quête vers une "tension vers l'impossible », semblable à un désir simple et naturel de curiosité envers l'inconnu, et également le surgissement de ce territoire inconnu dans l'espace connu, comme une violation, un frottement spatial entre deux zones, dont l'une, imperceptible, se donne soudain à voir (Bayon, 2007: 88).

L'homme d'Anatomie de l'enfer contraint, au départ, de regarder ce qu'il ne devrait pas voir, finit par éprouver une curiosité mêlée de désir et de crainte pour ce corps « impossible». Quant à la femme, bien qu'elle n'ait aucune emprise sur ce corps qu'elle ne comprend pas, elle cherche néanmoins à l'explorer comme un sujet d'expérimentation scientifique. Mais la science n'est d'aucun secours tant le dégoût et l'incompréhension de soi dominent. L'héroïne d'Anatomie de l'enfer considère son corps comme une aberration de la nature. Son ressenti est très proche de celui du personnage masculin d'Orgie de Pasolini qui, ne pouvant comprendre l'altérité de la femme, méprise et insulte son corps :

\author{
Tu trouves naturel \\ d'avoir ce sexe, \\ cette frontière lisse \\ au fond du ventre \\ léchée par une marée noire... \\ Mais non ce n'est pas naturel... pas naturel! \\ Tu ne sais pas que c'est intolérable et scandaleux \\ de vérifier dans son propre cas la règle générale ? \\ Toi, en te montrant nue, c'est ce que tu fais... (Pasolini, $1988: 77$ ).
}

La femme, dans Orgie comme dans Anatomie de l'enfer, est dépossédée de son corps. Suintant et visqueux, il se présente comme un continent impénétrable même pour celle qui le possède. En cela Anatomie de l'enfer fait écho à Une vraie jeune fille, le premier film de la réalisatrice évoqué précédemment. Alice, l'adolescente du film de Breillat expose ses seins, son ventre et même ses poils pubiens que l'on voit, à plusieurs reprises, dépasser de sa culotte. Elle ne cherche pas, contrairement aux adolescentes de 36 fillette et d'À ma sœur, à perdre sa virginité mais juste à comprendre les mécanismes de ce corps en pleine éclosion. Les garçons qui l'entourent n'auront pas accès à cette féminité que l'homosexuel d'Anatomie de l'enfer explorera dans ses moindres recoins, en allant jusqu'à boire, dans une sorte de parodie de rite initiatique, le sang des règles de celle qui se considère comme son ennemie. Anatomie de l'enfer se présente comme la parfaite synthèse de tous les films de son auteur. Face à un sexe qui le terrorise, l'homme, chez Breillat, n'arrive plus à jouir. Dans Parfait amour! et Romance, les personnages masculins n'ont pas d'érection, ils ne peuvent pas satisfaire leurs compagnes alors que dans Sex is comedy, un acteur, visiblement complexé, cherche à avoir la plus grosse prothèse pénienne possible afin de pouvoir mieux simuler l'acte sexuel.

Si les femmes, chez Breillat, sont meurtries au sein de leur chair, les hommes, quand ils ne sont pas impuissants, sont réduits à leurs seuls attributs, que l'on songe au plan furtif d'une fellation pratiquée sur un sexe au corps invisible dans Parfait 
amour ! ou à la séquence de «l'enfer » dans Romance au cours de laquelle des sexes de hardeurs éjaculent sur le pubis du personnage interprété par Caroline Ducey. La thématique de la recherche de la jouissance qui d'Une vraie jeune fille à Romance, traverse l'œuvre de Breillat, semble avoir totalement disparue d'Anatomie de l'enfer. La femme ne prend plus aucun plaisir à avoir des relations sexuelles alors que l'homme fait l'amour, de manière mécanique, comme pour éprouver une masculinité qu'il craint de ne voir à jamais disparaître. L'homosexuel d'Anatomie de l'enfer pénètre un corps qui le répugne. Il ne cherche pas à jouir, contrairement aux personnages qu'a pu interpréter Rocco Siffredi lorsqu'il tournait encore dans des films classés X. Dans la pornographie straight, à laquelle a longtemps participé Siffredi, l'excitation est suscitée dans un dispositif où les codes de représentations respectent la virilité. Les érections perdurent et les éjaculations se multiplient sans qu'il n'y ait jamais un quelconque temps de repos. Breillat déconstruit ces modes de représentations en en interrogeant les fondements. Si le sexe est surreprésenté, il n'a rien plus rien d'excitant surtout pour les amateurs de X mainstream.

Dans Anatomie de l'enfer, la notion de désir tend totalement à s'estomper. Le spectateur est appelé à contempler le corps de la femme tel qu'il est réellement et non comme le présente l'industrie de la pornographie contemporaine. Quand elle n'est pas simplement habillée (jean et tee-shirt blanc), la femme est entièrement nue, le pubis et les aisselles poilues. Or si le film de Breillat fait écho à l'univers de la pornographie, c'est plus dans le cinéma $X$ des années 80 qu'il faut aller puiser ses références. À cette époque, les sexes féminins n'étaient pas rasés et les sécrétions que les nouveaux pornographes ont aujourd'hui tendance à occulter (glycine, sang) étaient représentées sans fausse pruderie. Breillat occulte la dimension fantasmatique du porno chic en filmant, de manière frontale, le corps et les organes sexuels de ses personnages. Loin du glamour revendiqué par un certain type de production pornographique, Breillat expose des corps sales et salis, à l'image de ses lèvres vaginales maquillées à grands coups de rouge à lèvres et de ce sexe ensanglanté mais sans cesse pénétré. À travers ces plans, bien plus risibles qu'érotiques, Breillat parodie une certaine tendance du cinéma pornographique contemporain. Après avoir peinturluré le sexe d'Amira Casar, Rocco Siffredi la maquille comme un clown en faisant délibérément déborder le rouge à lèvres de sa bouche. La femme se présente, dès lors, comme la version dégradée des starlettes sur-maquillées du X mainstream. Le sexe poilue et maquillée de l'héroïne d'Anatomie de l'enfer s'opposent aux corps lisses des bimbos siliconées de la pornographie traditionnelle. Ce parti-pris de laideur renvoie aux réflexions de Bataille qui, dans L'Érotisme, refusait d'incorporer l'idée de beauté à la notion d'érotisme :

Parlant de la beauté d'une femme, j'éviterai de parler généralement de la beauté. Je veux seulement saisir et limiter le rôle de la beauté dans l'érotisme. Sous une forme élémentaire, il est à la rigueur possible d'admettre une action, dans la vie sexuelle des oiseaux, des plumages multicolores et des chants (Bataille, 1957 : 151). 
Pour Bataille, comme pour Breillat, l'érotisme est bien plus pulsionnel qu'esthétique. Les organes génitaux, contrairement à ceux rasés et maquillés que le cinéma X ne cessent de mettre en valeur, n'ont rien d'attirant. Malgré la crudité de certains plans, Anatomie de l'enfer ne peut être considéré comme un film pornographique puisqu'il ne cherche, à aucun moment, à exciter le spectateur. À la pénétration habituelle, infiniment reprise et répétée dans le cadre d'une pornographie traditionnelle, la réalisatrice d'Anatomie de l'enfer oppose la dissolution du membre masculin dans la femme. Ce n'est plus le pénis qui force le vagin mais le vagin qui absorbe le pénis. Le sexe féminin, réduit au rang de gouffre, n'est pas mis en valeur de la même manière que dans le cinéma $X$ traditionnel. Breillat montre ici ce que le cinéma, hors-porno, cache. Elle met en scène, le regard que les femmes portent sur leur propre corps tel que le décrit Elisa Brune dans La Révolution du plaisir féminin :

De manière générale, les femmes n'aiment pas leur sexe. Elles ne trouvent pas ça beau. Dans toute la culture, il n'y a pas une seule représentation de vulve complète. Soit on est dans le porno, soit il n'y a pas de représentation (Brune, $2012: 161$ ).

L'héroïne d'Anatomie de l'enfer, bien qu'elle méprise son corps, ne le rejette pas. Elle en a fait une arme grâce à laquelle elle tient les hommes sous sa coupe. L'homosexuel du film de Breillat, même s'il ne supporte pas la vue de ce sexe grumeleux pareil au coup d'un poulet plumé, lui envie son obscénité. Fantasmant sur un corps qu'il ne désire pas, alors que les autres hommes ont en horreur ce corps qu'ils désirent, le personnage incarné par Rocco Siffredi met sur le même plan passion et aversion. À la fin du film, son dégoût pour ce sexe qui n'est pas le sien se métamorphose en véritable haine du genre féminin. Dans un bar, face à un pilier de comptoir, l'homosexuel devenu, en l'espace de cinq nuits, une caricature d'hétérosexuel machiste, emploiera des termes très crus pour parler de cette femme qui s'est abandonnée à son regard. À cet instant, le Siffredi acteur de Breillat est redevenu le Siffredi super-star du X. Le regard qu'il porte sur les femmes est d'ailleurs semblable ici à celui des acteurs de films pornographiques. Réduite au rang de créature hypersexuée prenant plaisir à être avilie, la femme ne peut cependant pas être considérée comme une simple victime. Breillat, à l'image de certaines militantes porno-féministes, réutilise le discours misogyne du X maintsream pour mieux le subvertir. Les insultes les plus courantes (salope, pute) sont une manière pour ces nouvelles travailleuses du sexe d'affirmer leur droit au plaisir et à la sexualité. Dans Le Discours pornographique, Marie-Anne Paveau parle même d'une véritable réappropriation féministe de termes tels que Slut et Whore:

J'aborde ces termes sous leur forme anglaise car ils sont utilisés tels quels dans les textes et réalisations francophones. Les mots slut et whore, respectivement "salope » et "pute », sont des appellations très importantes dans le mouvement sex-positif car elles font l'objet de ce que Judith Butler et d'autres théoriciennes des études de genres appellent reappropriation ( « réappropriation ») ou reclamation (du verbe to reclaim, « revendiquer »), qui sont des formes de résignation. Il existe en effet chez les militantes et militants féministes, queer, et dans le mouvement LGBTIQA en général, une 
volonté de reprendre les termes stigmatisants de l'adversaire pour en faire des outils de lutte (Paveau, 2014 : 116).

Dans Anatomie de l'enfer, la femme ne se désigne pas en des termes aussi vulgaires. Bien qu'elle revendique, à l'image de la majorité des héroïnes de Breillat, sa propre perversité, elle ne cherche pas à l'ancrer dans un quelconque discours antipatriarcal. Si Breillat se dit féministe, elle refuse que ses films soient étiquetés de la sorte. En mars 2001, dans une interview pour L'Humanité, la réalisatrice de Romance avait exprimé toute son aversion pour le cinéma féministe qui, selon elle, ne chercherait pas tant à défendre la femme qu'à l'aliéner encore plus :

Oui, résolument. Seulement je ne veux pas que mes films soient étiquetés «féministes », car le cinéma c'est d'abord un art. Je déteste les films qui sont faits pour dénoncer quelque chose, comme ces films féministes qui combattent la pornographie au nom de la dignité de la femme. Malheureusement, ils sont d'emblée dans le même camp que ceux qui asservissent les femmes, au nom de cette même dignité. Dans Une vraie jeune fille, je montre la sexualité naissante, la transformation du corps d'une jeune fille, ce qui pourrait être épanouissant. Mais on nous fait vivre ce bourgeonnement comme une honte. Ce que je ne savais pas encore lorsque j'ai tourné le film, c'est que les femmes, en intégrant l'inconscient collectif, ont fini par trouver du plaisir dans la honte, dans la soumission aux hommes (Breillat, Pätzold, 2001).

Si Marie, dans Romance, est initiée aux rapports sadomasochistes par un homme plus âgé, la femme d'Anatomie de l'enfer humilie l'homme, qu'elle paye pour la regarder, en l'exposant à ses plus bas instincts. Dans les deux cas, c'est la femme qui revendique son désir qu'il soit sadique ou masochiste. Le cinéma de Breillat est un cinéma de l'ambiguïté. La femme y assouvit ses désirs tout en y étant contrainte. Le regard qu'elle porte sur sa sexualité est extérieur, comme prisonnier d'un imaginaire de la soumission contre lequel il ne semble pas possible de lutter. À la fin d'Anatomie de l'enfer, l'homme pousse la femme, au-dessus d'une falaise, dans une étrange scène teintée d'onirisme. A cet instant, il est impossible pour le spectateur de savoir d'où proviennent réellement ces plans. S'agit-il de la réalité ou d'un fantasme imputable au personnage de Rocco Siffredi ?

Breillat laisse planer le doute comme pour mieux désarçonner son public. Elle met en scène ici un fantasme de réification totale du corps de la femme. Au début entière et habillée, puis nue et enfin réduite à son seule sexe, l'hérö̈ne d'Anatomie de l'enfer finit par disparaître. Alors qu'elle apparaît, dans un premier temps, comme un modèle pictural semblable, dans sa nudité, à La Maja desnuda de Goya, son sexe, progressivement placé au centre même de l'image, renvoie inlassablement à L'Origine du monde de Courbet. La femme d'Anatomie de l'enfer, en exhibant ce corps qu'elle méprise mais dont elle reconnait la toute puissance, esquisse un léger sourire semblable à celui de la Maja du tableau de Goya. Elle s'amuse de l'effet qu'elle produit sur un homme qui découvre, pour la première fois, le corps d'une femme dans son intégralité. Pour Peggy Sastre, auteure de Ex Utéro-Pour en finir avec le féminisme, la scandaleuse de Breillat est synonyme d'effacement abyssal (Sastre, 2009: 97). C'est parce qu'elle domine l'homme, en se soumettant 
paradoxalement à lui, que la femme d'Anatomie de l'enfer est mise à mort, que cela soit réel, sur le plan diégétique, ou simplement figuré, sur le plan symbolique. Comme Alice, la jeune fille perverse et manipulatrice de Glissements progressifs du plaisir d'Alain Robbe-Grillet, l'héroïne d'Anatomie de l'enfer feint la soumission, notamment sur le plan sexuel, pour mieux arriver à ses fins. L'homme, au départ troublé par ce corps qu'il ne connaît pas, finit par en prendre possession. Assauts nocturnes, sodomie avec une bêché, marques au rouge à lèvres sur le sexe : tous les fantasmes semblent permis au sein de cet espace blanc immaculé qui n'est pas sans évoquer le château de Silling des 120 journées de Sodome de Sade. Il n'est d'ailleurs pas anodin que la structure cyclique du film de Breillat rappelle celle du roman Sadien. Le récit est, en effet, découpé en cinq nuit au cours desquelles l'homme pousse, à chaque fois, un peu plus loin, son exploration de l'anatomie féminine.

Moins radicale que le Divin Marquis, la cinéaste utilise néanmoins un même procédé de gradation. Au fil des jours et des nuits, les perversions s'intensifient pour mieux entraîner les personnages dans l'oubli quand ce n'est dans la mort. Cependant, alors que chez l'auteur des 120 journées de Sodome, les hommes et les femmes sont mis sur un même plan d'égalité, dans le cinéma de Breillat, seule la gent féminine est appelée à connaître un destin tragique, Que l'on songe à Elena et Anaïs, l'une tuée, l'autre violée par un détraqué sexuel dans À ma sœur ou à Frédérique, assassinée à coup de couteau, par son amant, dans Parfait amour!, il n'y a jamais de sexualité féminine épanouie dans l'œuvre de la réalisatrice d'Anatomie de l'enfer. La femme, réduite à son seul corps, ne peut sortir de sa condition. Breillat ne met pas en scène l'émancipation sexuelle de la femme puisque celle-ci, toujours bornée, au regard des autres, n'arrive jamais à s'accomplir. Le corps féminin est autant un fléau pour la femme que pour l'homme.

En jouant avec les codes de la représentation pornographique et en incorporant, au sein de son œuvre, toutes sortes de références culturelles et mythologiques, Breillat met en scène une image de la femme d'une infinie complexité. Le corps féminin, bien qu'il puisse être considéré comme un arme permettant aux femmes d'asservir tous les hommes, même homosexuels, si l'on évoque le cas particulier du personnage masculin d'Anatomie de l'enfer, est appelé à être détruit. Il est, au sens propre comme au sens figuré, une véritable « anatomie de l'enfer ».

\section{RÉFÉRENCES BIBLIOGRAPHIQUES}

Bataille, G., (1957) L'Érotisme. Paris, Minuit.

Bataille, G., (1993) Histoire de l'œil. Paris, Gallimard.

Bayon, E., (2007) Le cinéma obscène. Paris, L'Harmattan.

Breillat, C., Pätzold, B., (2001) «Catherine Breillat balaie les tabous» in L'Humanité.fr [en ligne]. Disponible sur : http://www.humanite.fr/node/244000 [Dernier accès le 11/11/2015].

Breillat, C., (2001) Pornocratie. Paris, Denoël. 
Brune, E., (2012) La Révolution du plaisir féminin. Paris, Odile Jacob.

Duras, M., (1982) La Maladie de la mort. Paris, Minuit.

Fouquette, A., (1995) Il y a deux sexes. Essais de féminologie. Paris, Gallimard.

Leiris, M., (1939) L'Âge d'homme. Paris, Gallimard.

Pasolini, P.P., (1988) Orgie. Paris, Acte Sud.

Paveau, M.A., (2014) Le Discours pornographique. Paris, La Musardine.

Regard, F., (2002) L'écriture féminine en Angleterre. Paris, PUF.

Sastre, P., (2009) Ex Utéro-Pour en finir avec le féminisme. Paris, La Musardine.

Stevens, S., (2013) «Pour en finir avec l'obscénité féminine: mythes sexuels et politiques érotiques dans Pornocratie de Catherine Breillat» in Oktapoda, O. (éd.), Mythes et érotismes dans les littératures et les cultures francophones de l'extrême contemporain. Amsterdam, Rodopi, pp. 77-95.

Vasse, D., (2004) Catherine Breillat: un cinéma du rite et de la transgression. Paris, Editions Complexe. 